\title{
Nondestructive Method for Measuring Dielectric Constant of Sheet Materials
}

\author{
Dhirendra Mathur \\ ECE Department \\ Govt. Engg. College Ajmer, INDIA \\ Email: dhirendra_mathur@yahoo.com \\ S. K. Bhatnagar \\ ECE Department \\ Jaipur Engg. College, Jaipur, INDIA \\ Email: bhatnagar_skb@yahoo.de
}

\author{
Vineet Sahula, Senior member IEEE \\ Dept. of ECE \\ NIT Jaipur, INDIA \\ Email: sahula@ieee.org
}

\begin{abstract}
In many applications including RF, Microwave dielectric constant $\left(\varepsilon_{r}\right)$ of the material is a key design parameter. Present methods for measurement of $\varepsilon_{r}$ are time-consuming and require a sample piece of the material on which some processing has to be done (destructive methods). This paper presents a nondestructive and simple method for determining $\varepsilon_{r}$ of sheet dielectric materials. The method is nondestructive in the sense that cutting of the material or any processing on it is not required. In this method resonant frequency $\left(f_{r}\right)$ of a pre-fabricated antenna is first measured with a known material and then again measured with the Material Under Test (MUT) as dielectric part of the microstrip antenna. The parameter $\varepsilon_{r}$ is then determined from the ratio of these resonant frequencies and the thickness of MUT. The experimentation is arranged so that errors due to measurement in patch dimensions and electrical connections to the feed line are eliminated. The method can be used for on-line quality control during production of dielectric sheets. An aperture coupled microstrip antenna has been designed and fabricated with $f_{r}=$ 8.4 GHz. A simple jig has been constructed to hold the antenna and connecting cable. It provides an easy means for changing the MUT and brings different areas under the patch. Measurements validate the design and simulation results.
\end{abstract}

Index Terms-Dielectric constant; measurement; aperture coupled; patch antenna; nondestructive method

\section{INTRODUCTION}

Modern wireless communication systems demand miniaturized, ultra wide band antenna structures that are preferably resonant at several frequencies. Optimization of such SystemOn-Package structures has led to heterogeneous dielectric medium whose dielectric constant $\left(\varepsilon_{r}\right)$ is engineered to meet performance specifications and might consist of layers of different dielectric materials (ceramic, foam, plastic, air etc.) of different thicknesses. Additionally a number of via holes and/or cavities of different dimensions may be embedded in the structure for engineering the effective dielectric constant. In such a medium the dielectric constant may vary from location to location. For good design of antenna and related circuits it is important to know accurate value of the dielectric constant of the substrate. Stripline, Patch, Ring and Cavity resonators have been extensively used for estimating substrate dielectric constant at microwave frequencies. However these estimations suffer from errors due to (i) variations in electrical and physical dimensions of the resonators due to fringing fields and (ii) discontinuities due to connectors/connections from the microwave/RF source. A large number of previously reported methods require fabrication of some resonant structure on the specimen Material-Under-Test (MUT) for measurements and estimation of dielectric constant. To overcome these problems and limitations, a new method has been proposed and validated in this paper.

\section{PREvious WORK}

A number of methods [1-16] are available for measuring the dielectric constant of a slab/sheet/laminate substrate material at microwave frequencies. These may be classified as either nondestructive or destructive methods. Irrespective of whether the method is destructive or nondestructive, the common technique is to use Cavity, Stripline, Patch or Ring resonators on the material under test or standard substrate for measurement of resonance frequency or other parameter for estimating substrate dielectric constant. Howell [1] has formed a closed edge resonance cavity by metalizing the substrate on all sides and determined resonance frequency for computing the dielectric constant. The method is a variant of the one suggested by Napoli \& Huges [2] It gives better accuracy but requires accurate measurement of length and frequency. However, the method is of destructive type and uses silver painting of the edges which adds cost. Itoh [3] has presented a nondestructive method of measuring the dielectric constant and loss factor of a slab type material using microstrip cavity with accuracy better than 3\% for dielectric constant and moderate accuracy for dissipation factor. The method is based on measuring resonance frequency and $\mathrm{Q}$ factor of microstrip cavity for two different situations when loaded with a known reference slab and then with the unknown material. The method has following limitations.

1) considerable amount of pressure is required (on the dielectric plate) for minimizing air gap between plate and the substrate.

2) the method is not suitable for crystalline and ceramic materials.

3) the method uses complex equations for various computations involved and a computer support is essential for solving them.

In a recent paper Yao et al. [4] has used cavity perturbation method to measure permittivity of dielectric sheet by measuring shift of frequency due to the presence of sample dielectric. Accuracy less than $3.8 \%$ in the case of rectangular cavity 
whereas less than $0.4 \%$ in the case of cylindrical cavity is achieved. However, the method requires small piece of sample so that frequency shift is less than 10\%. Dankov [5], [6] has presented method to measure dielectric anisotropy of a disk sample in a multilayer resonance cavity structure based on $T E_{011}$ and $T M_{010}$ modes. The method is especially suitable for multilayer materials e.g. bonded substrates, composite antenna radomes, film \& tape structures etc. in one-, two-, and threelayers. Dielectric constant of unknown dielectric layer can be extracted if the same is known for the other layers. The errors in measurements decrease with decreasing thickness of samples. Murray Olyphant [7] has presented strip-line method of measuring effective dielectric constant of copper-clad laminates using microstrip circuits e.g band-pass or band-stop filters. Precision of measurement is limited by the precision of strip length and frequency measurements. Air gap appears to be the major limitation on accuracy. Das, Voda and Pozar [8] have presented two measurements methods (i) method using two-microstrip-line and (ii) method using two-layer stripline of dielectric constant of microwave substrates with accuracy of $0.5-1.0$ percent for the first method and 0.4 percent for the second. The second one is a nondestructive method and uses a standard substrate with a $50 \mathrm{ohm}$ microstrip line connected to a network analyzer by two connectors at the ends. The test substrate is etched to remove copper from one side and cut into rectangular shape with width greater than two wavelengths. The test substrate is used to cover a portion of the microstrip line and is clamped from top to bottom. The entire structure is cascade constructing of stripline, between two microstrip lines. The method has following limitations- (i) Air gap between standard and test substrate is a source of error (ii) Dielectric constant of the test substrate should be close to that of the standard substrate and also the thicknesses of the two should be close to each other. Pannell and Jervis [9] have presented (i) Reflection cancellation and (ii) Line balancing methods for the measurement of dielectric constant using microstrip line. The methods give value of dielectric constant beneath the microstrip line i.e. specific to the length of microstrip line in question. The measurement errors are mainly attributed to the discontinuity at the coaxial line to microstrip transition, yet accuracy within $8 \%$ is reported. Carbonini et al. [10] have presented a method to estimate transverse dielectric constant of laminated microwave materials. The method requires samples to be cut in rectangular shape with cladding removed before inserting in a waveguide. Phase difference between the unloaded and loaded test set up is measured to obtain dielectric constant. Sarhan et al. [11] have measured dielectric constant of FR-4 substrate using a band-pass filter. Slawomir and Zaradny [12] have measured dielectric constant of a microwave laminate using symmetricstrip-transmission line technique with accuracy less than $3 \%$. Yehuda Kantor [13] has presented three methods- microstrip resonator, stripline resonator and closed disk resonator to determine dielectric constant of laminates having thickness of $0.2-3 \mathrm{~mm}$ with dielectric constant varying from 2 to 20 . Du Shimin [14] has presented a method of measuring dielectric constant of a thin slab material using resonance frequency of a microstrip patch antenna. Results have been compared with standard cavity resonator method. The method requires constructing a rectangular patch antenna on the substrate and measuring input impedance using network analyzer. Resonance frequency is found from the input impedance and phase characteristics. Relative permittivity of the substrate is obtained using analytical equations. The method has following limitations.

1) The method may not be truly nondestructive as it requires fabrication of patch antenna on the test sample

2) Estimation of dielectric constant requires accurate measurement of patch geometry

3) The method requires substrate to be 3-4 times larger in size than patch having thickness much less than $1 / \beta$

4) Computation complexity is higher.

Singh, De and Yadava [15] have presented a nondestructive method of measuring dielectric constant of a slab material with accuracy of $1.6 \%$ and $1.9 \%$ for high permittivity $\left(\varepsilon_{r}=10.2\right)$ substrates and $4.5 \%$ for low permittivity $\left(\varepsilon_{r}=2.22\right)$ substrates. The method has following limitations.

1) The method may not be truly nondestructive as it requires fabrication of patch antenna on the test sample

2) Accuracy depends on precise measurement of patch dimensions.

3) The method requires correction factor for accurate measurement of patch dimension, correction factor for fringing effect and fringing capacitance. Uncertainty in the manufacturer's specified value and uncertainty in the measurement of dielectric constant affects the results.

Verma et al. [16] have also used patch resonator to determine dielectric constant of substrates in single layer and threelayers. The method is based on accurate determination of resonance frequency of microstrip patch using algorithm based on modified Wolff model (MWM) to extract value of dielectric permittivity using measured frequency, substrate thickness and accurate dimension of the patch. For the aforesaid methods and techniques of dielectric constant measurement, following limitations and sources of errors are observed.

1) Air gap between sample piece and standard substrate affects the accuracy of measurement of resonance frequency which leads to inaccuracies in estimation of dielectric constant.Accurate estimation of dielectric constant is based on accurate measurement of resonator dimensions.

2) Most of the methods are of destructive type, except [3], [8]. However, these two methods also require rectangular sample in appropriate size. A destructive type of method may not be recommended for costly substrates.

3) Some of the methods use specific size and type of dielectric samples.

4) The computational time complexity of aforesaid methods is high requiring computer support.

5) A large number of fabrication steps as well as a complex test set up is required making measurements time consuming. 
The outline of rest of the paper is as follows. Section III proposes a method which is non-destructive type. Section IV presents design of aperture coupled patch antenna. Simulation results obtained using electro-magnetic solver Zeland's IE3D V14.0 are discussed. Details of fabrication and measurements are provided in Section V. Results and discussions are given in Section VI followed by conclusions.

\section{Our Proposed Method}

The new proposed method is nondestructive, quick in estimation and simple in use. It uses a multi-dielectric aperture coupled antenna as patch resonator with an air dielectric layer between patch and ground plane. The air layer enables easy insertion of dielectric sheet sample of any size beneath patch layer. Thus the air dielectric layer and dielectric test material become part of the structure and measurement setup. There is no restriction on the type of dielectric sheet material as long as its thickness is less than the space available for insertion. The method simply requires measurement of resonance frequencies $f_{r e_{1}}$ and $f_{r e_{2}}$ with reference material and material under test (MUT) respectively using a network analyzer. Estimation of dielectric constant is independent of patch geometry hence related inaccuracies are avoided. The method is suitable for substrate size even equal to that of patch size.

The basic theory of the proposed method is as follows. An aperture coupled microstrip antenna is designed and fabricated for the frequency at which $\varepsilon_{r}$ is to be measured. The antenna dielectric consists of three layers. First layer $\left(\varepsilon_{r}=\varepsilon_{r p}\right)$ of thickness $h_{p}$ physically supports the patch. Third layer $\left(\varepsilon_{r}=\varepsilon_{m u t}\right)$ of thickness $h_{m u t}$ is placed on the ground plane of the antenna. The middle layer (air) of thickness $h_{\text {air }}$ fills the space between the first layer and the third layer. Composite Dielectric Constant $\varepsilon_{c}$ of this 3-layer structure is given in [17], [18] and reproduced in (1).

$$
\frac{h_{t}}{\varepsilon_{c}}=\frac{h_{m u t}}{\varepsilon_{r_{\text {mut }}}}+\frac{h_{\text {air }}}{\varepsilon_{r_{\text {air }}}}+\frac{h_{p}}{\varepsilon_{r p}}
$$

Let's consider following known microstrip antenna design formulae.

$$
\begin{aligned}
W & =\frac{c}{2 f_{r}} \sqrt{\frac{2}{\varepsilon_{c}+1}} \\
f_{r} & =\frac{c}{2(L+2 \Delta L) \sqrt{\varepsilon_{r e}}} \\
\varepsilon_{r e} & =0.5\left(\varepsilon_{r}+1\right)+0.5\left(\varepsilon_{r}-1\right)(1+12 h / W)^{-0.5}
\end{aligned}
$$

Let there be two patch antennas differing only in dielectric constant of antenna substrate material (having same patch dimensions (L, W) and substrate thickness (h). If the change in $\varepsilon_{c}$ is small and all other parameters are held constant, then the effect of fringing field can also be assumed to be constant for the two antennas. Using above relations it can be shown that

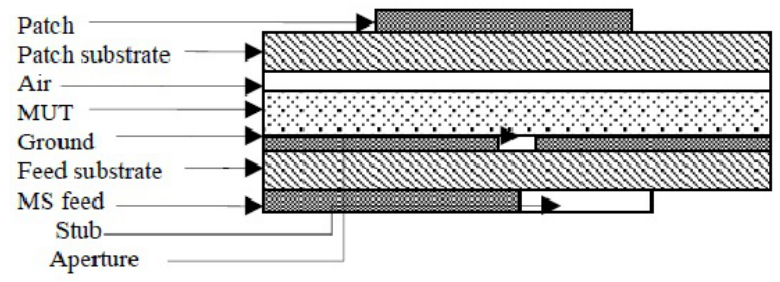

Fig. 1. Aperture coupled microstrip antenna with multilayer dielectric structure, dimensions (in mm): $\mathrm{L}_{p}=11.01, \mathrm{~W}_{p}=15.03, \mathrm{~W}_{m s}=1.98, \mathrm{~L}_{s t u b}=2.55$, $\mathrm{X}_{\text {aperture }}=0.97, \mathrm{Y}_{\text {aperture }}=6.5, \varepsilon_{r_{\text {patch }}}=\varepsilon_{r_{\text {feed }}} 3.2$, thickness $=0.75, \mathrm{~h}_{\text {total }}=1.84$. Dimensions of aperture and stub are optimized for MUT of $\varepsilon_{r}=2.2$

$$
\varepsilon_{r e_{2}}=\varepsilon_{r e_{1}}\left(\frac{f_{r_{1}}}{f_{r_{2}}}\right)^{2} .
$$

Here, $\varepsilon_{r e_{1}}$ and $f_{r_{1}}$ are effective dielectric constant and resonance frequency respectively for the patch antenna with first dielectric medium with known dielectric constant (reference substrate) while $\varepsilon_{r e_{2}}$ and $f_{r_{2}}$ refer to second dielectric medium whose dielectric constant is to be estimated. The effective dielectric constant $\varepsilon_{r e_{2}}$ can be found using the relation in 5, if $f_{r_{2}}$ is known. Then using (4) $\varepsilon_{r}$ can be found. For the multi-dielectric layer structure as shown in Fig. 1, (4) shall be modified as:

$$
\varepsilon_{c e}=0.5\left(\varepsilon_{c}+1\right)+0.5\left(\varepsilon_{c}-1\right)(1+12 h / W)^{-0.5} .
$$

Here, $\varepsilon_{c e}$ refers to effective dielectric constant of composite medium and $\varepsilon_{c}$ is the composite dielectric constant of the multilayer dielectric medium. Dielectric constant of the unknown material (MUT) is determined following these steps.

(a) Design the multilayer aperture coupled antenna with known reference dielectric layer as MUT (b) Design and make an appropriate jig to mount the antenna. (c) Obtain fr1from return loss plot and find $\varepsilon_{r e 1}$ using (4) (d) Replace reference dielectric layer with the unknown MUT and find $f_{r_{2}}$ (e) Using (1), (5) \& (6) find $\varepsilon_{r e_{2}}, \varepsilon_{c}, \varepsilon_{r_{m u t}}$ respectively. (f) Repeat the steps (d) \& (e) for other MUTs.

For better accuracy it is important to select the reference dielectric material such that resonance frequency $f_{r_{1}}$ is close to that using MUT, which means effective dielectric constant of the dielectric structure with MUT should differ only marginally with that using reference substrate.

\section{Design \& Simulation}

The proposed aperture coupled microstrip antenna with multidielectric structure as designed is shown in Fig. 1.

There are three dielectric layers above ground plane viz. material under test (MUT), middle layer of air and patch substrate on top. By having an air dielectric layer in middle gives two benefits. One, it facilitates insertion of MUT and secondly, the variation of effective dielectric constant can be kept much smaller compared to a large variation in $\varepsilon_{r}$ of MUT. This is a primary requirement for (5) to be valid. Simulations 
TABLE I

SIMULATION RESULTS

\begin{tabular}{|c|c|c|c|c|c|}
\hline MUT & $\varepsilon_{r}$ & $\mathrm{~h}$ & \multicolumn{2}{|c|}{ Calculated } & \multirow{2}{*}{$f_{r_{\text {sim }}}$} \\
\cline { 4 - 5 } & & & $\varepsilon_{c}$ & $\varepsilon_{c e}$ & \\
\hline \hline Diel.1(D1) & 2.2 & 0.39 & 1.66 & 1.54 & 8.4 \\
\hline Diel.2(D2) & 2.33 & 0.49 & 1.76 & 1.62 & 8.16 \\
\hline Diel.3(D3) & 3.0 & 0.24 & 1.58 & 1.47 & 8.56 \\
\hline Diel.4(D4) & 3.2 & 0.75 & 2.28 & 2.04 & 7.2 \\
\hline Diel.5(D5) & 9.4 & 0.64 & 2.45 & 2.18 & 6.96 \\
\hline
\end{tabular}

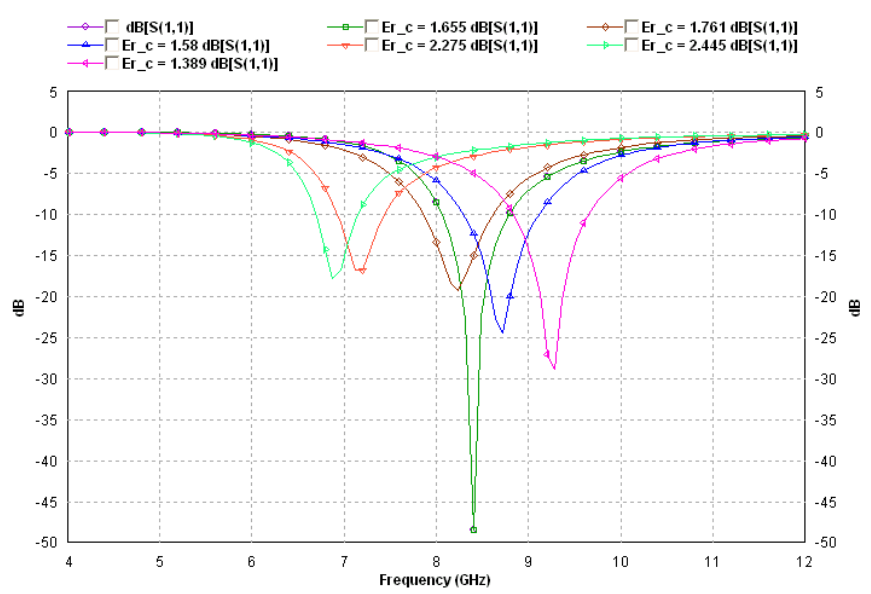

Fig. 2. Combined return loss plot for different MUTs

have been carried out on IE3D. Results are presented in Table II. Specified values of $\varepsilon_{r}$ for the sample MUTs are 2.2, 2.33, $3.0,3.2$, and 9.4 with total variation of 7.2. In the proposed method this variation has been reduced to 0.79 by cleverly including an air layer in the antenna dielectric. Variation in effective dielectric constant $\left(\varepsilon_{c e}\right)$ is even smaller (0.64) resulting in variation of resonance frequency from $6.96 \mathrm{GHz}$ to $8.56 \mathrm{GHz}$ only. Effective dielectric constant $\left(\varepsilon_{c e}\right)$ values or $f_{r_{\text {sim }}}$ suggests two distinct groups of dielectric samples viz. D1, D2, D3 and D3, D4 which means there should be at least two different reference dielectric mediums for measurements on the given set of five samples. Fig. 2 shows a combined plot of return loss for different MUTs.

It is observed that proper reference dielectric substrate yield $\varepsilon_{r_{m u t}}$ with good accuracy.

\section{FABRicAtion AND MEASUREMEnts}

Antenna structure has been fabricated as mentioned in Fig. 1. Present antenna was designed for $8.4 \mathrm{GHz}$ resonant frequency

TABLE II

EXTRACTED $\varepsilon_{r}$ FOR MUT

\begin{tabular}{|c|c|c|}
\hline MUT & $\varepsilon_{r}$ & $\varepsilon_{r_{\text {mut }}}$ \\
\hline \hline D1 & 2.2 & 2.152 \\
\hline D2 & 2.33 & 2,331 \\
\hline D3 & 3.0 & 2,997 \\
\hline D4 & 3.2 & 3.177 \\
\hline D5 & 9.4 & 9.62 \\
\hline
\end{tabular}

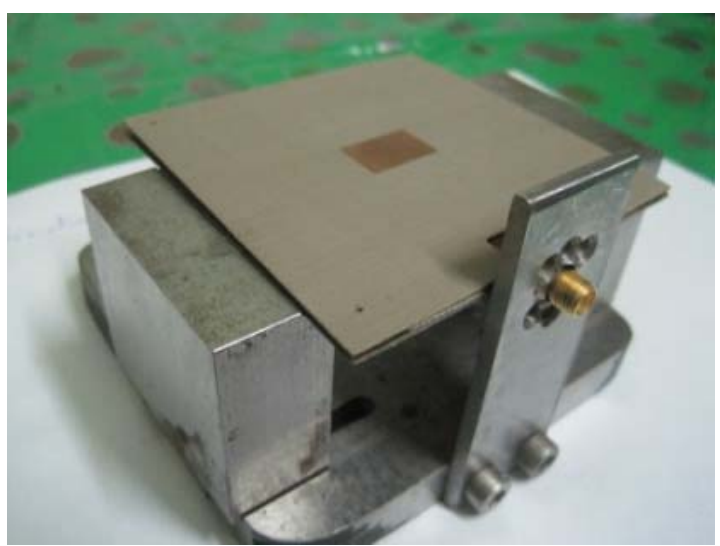

Fig. 3. Fabricated antenna and Jig Fixture

TABLE III

MEASUREMENT RESULTS

\begin{tabular}{|c|c|c|c|c|}
\hline MUT & $f_{r_{\text {meas }}}$ & $\varepsilon_{r_{\text {meas }}}$ & $\varepsilon^{*}$ \\
\hline \hline $\mathrm{D} 1$ & 8.16 & 2.47 & 2.2 \\
\hline $\mathrm{D} 2$ & 8 & 2.35 & 2.33 \\
\hline $\mathrm{D} 3$ & 8.4 & 2.91 & 3.0 \\
\hline & D4 & 7.56 & 2.74 & 3.2 \\
\cline { 2 - 5 } *Typical value of & \multicolumn{3}{|c|}{ as per manufacturer's data sheet }
\end{tabular}

but any other frequency can also be used. The jig consists of a mechanically stable stand. The aperture coupled microstrip antenna is firmly clamped on it. Feed line is suitably connected to a network analyzer for measuring $f_{r}$. The jig was made to properly hold the assembly of antenna with SMA connector so as to avoid lifting of substrate due to cable connecting PNA and antenna, thus keeping it flat and straight.

Distance between patch and ground plane is the total thickness of antenna's multi-layered dielectric. Design of the jig is such that it accurately maintains this distance during measurements and takes proper care to maintain centre-to-centre alignment of patch and aperture. Dimensions of jig are large enough so that all metal surfaces/edges are more than $\lambda / 2$ away from the patch. Photographs of the fabricated antenna and the jig are shown in Fig. 3. Measurements of return loss $S_{11}$ parameter for different MUTs were performed using Agilent PNA E8363B. Measurements were taken on several samples of four different dielectric materials. Measured results and extracted values of dielectric constants are given in Table III.

Measured values of dielectric constants have been compared with their values as given in data sheets. In manufacturing of dielectric sheets there is variation in $\varepsilon_{r}$ value from lot to lot therefore it can be concluded that the agreement between measured values and published values is good.

\section{RESUltS AND Discussion}

The proposed method is based on (5) therefore all the dimensions/parameters have to be selected such that (5) remains valid. Selection of feeding technique is crucial. Aperture 
coupling has been used to avoid problems associated with probe feeding and direct inset feeding. Entire measurement setup and the connections to network analyzer are stationary and are not disturbed during any series of measurements. Only resonant frequencies are measured experimentally and $\varepsilon_{r}$ is calculated from the ratio of these frequencies. Therefore any error associated with microwave connections cancel out. This increases accuracy and reliability of $\varepsilon_{r}$ value within the validity range of (5). The proposed method does not require cutting of sample pieces from the given material or fabrication of patch, ground or any other structure on, or any fabrication through the substrate MUT. Therefore it is nondestructive. The method is unique as the resonator dimensions are eliminated from $\varepsilon_{r}$ calculations and associated errors are also eliminated. The method is quick, simple and easy to use. It can be used during production of substrates. Dielectric constant $\varepsilon_{r}$ can be measured on a very small area, if required. Small measurement area improves scanning for quality control during production. The method can be used to estimate $\varepsilon_{r}$ at different places on a heterogeneous dielectric substrate where effective dielectric constant $\left(\varepsilon_{r}\right)$ may vary from place to place on the same substrate.

\section{CONCLUSIONS}

A new method for experimental measurement of dielectric constant has been proposed which is based on simple well known formulae of antenna design. The new method is nondestructive, quick, simple and easy to use. It only requires measurement of resonance frequency for different MUTs. It requires only few minutes to insert MUT in the air gap between patch substrate and ground plane and taking measurements of return loss. Effective dielectric constant is estimated from the simple relation of ratio of resonance frequencies which is free from physical dimensions of the patch resonator hence inaccuracies associated with measurement of physical dimensions of the patch, fringing field effect, microwave connectors etc. are also eliminated. Another novelty of the method is that it does not require fabrication of patch on the substrate under test and can be used to scan substrates to observe variation in from place to place in a substrate, from substrate to substrate in a batch and from batch to batch in a manufacturing environment. The proposed method can be used as a quality control tool for manufacturing sheets of dielectric material.

\section{ACKNOWLEDGMENTS}

The authors acknowledge with thanks the help received from the microwave and antenna group at Space Application Center, Ahmedabad, India for providing lab facilities for performing necessary measurements. S.K. Bhatnagar thanks the management of JEC Group of colleges for permission to publish these results.

\section{REFERENCES}

[1] J. Q. Howell, "A quick accurate method to measure the dielectric constant of microwave integrated-circuit substrates," IEEE Transactions on Microwave Theory and Techniques, pp. 142-143, MARCH 1973.

[2] L. S. Napoli and J. J. Hughes, "A simple technique for the accurate determination of the microwave dielectric constant for microwave integrated circuit substrates," IEEE Transactions on Microwave Theory and Techniques, pp. 664-665, JULY 1971.

[3] T. ITOH, "A new method for measuring properties of dielectric materials using a microstrip cavity," IEEE Transactions on Microwave Theory and Techniques, pp. 572-576, MAY 1974.

[4] L. Yao, Z. Ni, T. Liu, and F. Xiao, "Measuring permittivity of the dielectric sheet by two kinds of microwave cavities," in International conference on Anti-Counterfeiting Security and Identification in Communication (ASID),2010, 2010, pp. 91-93.

[5] P. I. Dankov, "Two-resonator method for measurement of dielectric anisotropy in multilayer samples," IEEE Transactions on Microwave Theory and Techniques, vol. 54, no. 4, pp. 1534-1544, APRIL 2006.

[6] P. I. Dankov and S. A. Ivanov, "Two-resonator method for measurement of dielectric anisotropy in multilayer thin films, substrates and antenna radomes," in 34th European Microwave Conference, 2004, pp. 753-756.

[7] J. Murray Olyphant and J. H. Ball, "Strip-line methods for dielectric measurements at microwave frequencies," IEEE Transactions on Electrical Insulation, no. 1, pp. 26-32, MARCH 1970.

[8] N. K. Das, S. M. Voda, and D. M. Pozar, "Two methods for the measurement of substrate dielectric constant," IEEE Transactions on Microwave Theory and Techniques, vol. MTT-35, no. 7, pp. 636-=641, July 1987.

[9] R. M. Pannel and B. W. Jervis, "Two simple methods for the measurement of the dielectric permittivity of low-loss microstrip substrates," IEEE Transactions on Microwave Theory and Techniques, vol. MTT-29, no. 4, pp. 383-386, APRIL 2081.

[10] L. Carbonini, E. Gallina, E. Pagana, and L. Volpi, "A new technique for estimating the transverse dielectric constant of laminated microwave materials," in Proceedings of the 36th European Microwave Conference, 2006.

[11] S. Yamacli, C. Ozdemir, and A. Akdagli, "A method for determining the dielectric constant of microwave pcb substrates," International Journal of Infrared and Milimeter Waves, vol. 29, no. 2, pp. 207-216, 2008.

[12] S. Gruszczynski and M. Zaradny, "A simple resonance method of measurement of dielectric constant of thin and intermediate thickness microwave laminates," in 15th International conference on Microwaves, Radar and Wireless Communicationa, 2004.

[13] Y. Kantor, "Dielectric constant measurement using printed circuit techniques at microwave frequencies," in Electrotechnical Conference, MELECON 98, 1998.

[14] D. Shimin, "A new method for measuring dielectric constant using the resonant frequency of a patch antenna," IEEE Transactions on Microwave Theory and Techniques, vol. MTT-34, no. 9, pp. 923-931, September 1986.

[15] R. Singh, A. de, and R. S. Yadava, "A simple method for measuring dielectric constant at microwave frequency," in CPEM'90 Digest, 1990, pp. 236-237.

[16] A. Verma, Nasimuddin, and A. Omar, "Microstrip resonator sensors for determination of complex permittivity of materials in sheet, liquid and paste forms," IEEE Transactions on Antennas and Propagation, vol. 152, no. 1, pp. 47-54, February 2005.

[17] B. K. Yeong J. Yoon, "A new formula for effective dielectric constant in multi-dielectric layer microstrip structure," in IEEE Conference on Electrical Performance of Electronic Packaging, 2000.

[18] W. K. W. Ali and S. H. A. Charchafchi, "Using equivalent dielectric constant to simplify the analysis of patch microstrip antenna with multi layer substrate," in IEEE Antennas and Propagation Society International Symposium, 1998. 